\title{
The Study on the Real-Time Measurement of Pipe Shape Applicable During Its Bending Process
}

\author{
Myung-Jin Chung \\ Dept. of Mechatronics Engineering, Korea Polytechnic University, Korea \\ mjchung@kpu.ac.kr
}

\begin{abstract}
The importance of piping technologies related to refrigerant, operating fluid, and lubricant is increasing according to growth of the industries such as plant, electric home appliances, aviation, and automotive. The elongation during the bending and curvature change by spring back effect after the bending are caused by characteristics of pipe material. The fault in assembling is occurred by shape change of pipe. So, real-time measurement of pipe shape is required in the bending process. In this study, head for real-time measurement of pipe shape during the pipe bending process in the bending machine is proposed, designed, and manufactured. Verification test of developed measurement head is conducted. Also developed pipe shape measurement head is adopted and tested in the pipe bending machine.
\end{abstract}

Keywords: Pipe shape, Bending process, Real-time measurement, Laser, Spring back

\section{Introduction}

The importance of piping technologies related to refrigerant, operating fluid, and lubricant is increasing according to growth of the industries such as plant, electric home appliances, aviation, and automotive. The elongation during the bending and curvature change by spring back effect after the bending are caused by characteristics of pipe material such as elasticity and plasticity. Hea [1] summarized regarding wrinkling instability at the intrados, wall thinning at the extrados, spring back phenomenon, cross-section deformation, forming limit via the analysis of bending characteristics and multiple defects in tube bending. Zhang [2] simulated to reveal the spring back laws by using ABAQUS in the bending and spring back processes of minor-diameter thick-walled tube. Mun [3] achieved the reduction of working time and improvement of production capacity by considering the spring back effect. Jeon [4] described the spring back effect by analysis and verified the analysis by experiment for circular tube bending. Song [5] used the combination scheme of explicit and implicit to predict the spring back in the tube bending process. Guo [6] proposed a spring back bending angle prediction model on the combination of error back propagation neural network and spline function to predict the spring back bending angle in V-die air bending process. Fault during assembling is occurred by change of pipe shape, which is caused by error cumulated during the bending process of pipe having many bending points [7]. So, real-time measurement of pipe shape is required in the bending process. The head for measurement of pipe shape is required in the pipe bending process using the bending machine. Chung [8] proposed the method for measurement of pipe shape but did not verify the availability. The

Article History:

Received (November 18, 2019), Review Result (December 28, 2019), Accepted (January 29, 2020) 
method and system for measurement of pipe shape is required in the pipe bending process using the bending machine. In this paper, head for measurement of pipe shape in the real-time is proposed by using the analysis of change of pipe shape and is verified with developed measurement head in the pipe bending machine.

\section{Effects by spring back of pipe after the bending process}

The elongation during the bending process and curvature change, which causes the change of the bending angle and vertex location, by spring-back effect after the bending process are caused by characteristics of pipe material such as elasticity and plasticity as shown in [Figure 1]. So, fault during assembling is occurred by change of pipe shape, which is caused by error accumulated during the bending process of pipe having many bending points.

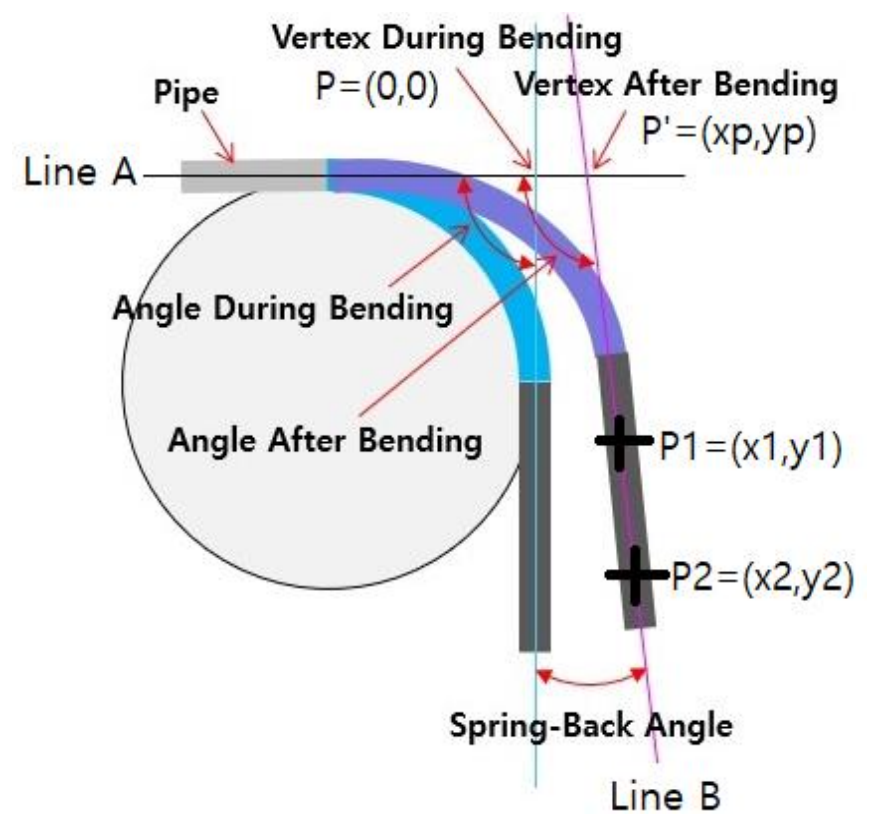

Figure 1. Change of pipe shape caused by spring back effect after bending process

\section{Method for measurement of pipe shape}

The change of bending angle and vertex location by measurement of pipe shape during and after bending process can be calculated. In [Figure 1], coordinates of point P1 and P2, which is center position of pipe, used to calculate the bending angle and vertex location. Image captured by camera and having spots made by line laser is processed to calculate the center point of the pipe as shown in [Figure 2].

\subsection{Bending angle}

Bending angle is calculated as angle made by line A, which is connecting the two centers of pipe before bending, and line $\mathrm{B}$, which is connecting the two centers of pipe after bending as Eq. (1).

$$
\theta=180^{\circ}-\tan ^{-1}\left(\frac{y 2-y 1}{x 2-x 1}\right)
$$


where, $\mathrm{x} 1$ and $\mathrm{y} 1$ are coordinates of measuring point $\mathrm{P} 1, \mathrm{x} 2$ and $\mathrm{y} 2$ are coordinates of measuring point $\mathrm{P} 2$. These points are selected as center of pipe.

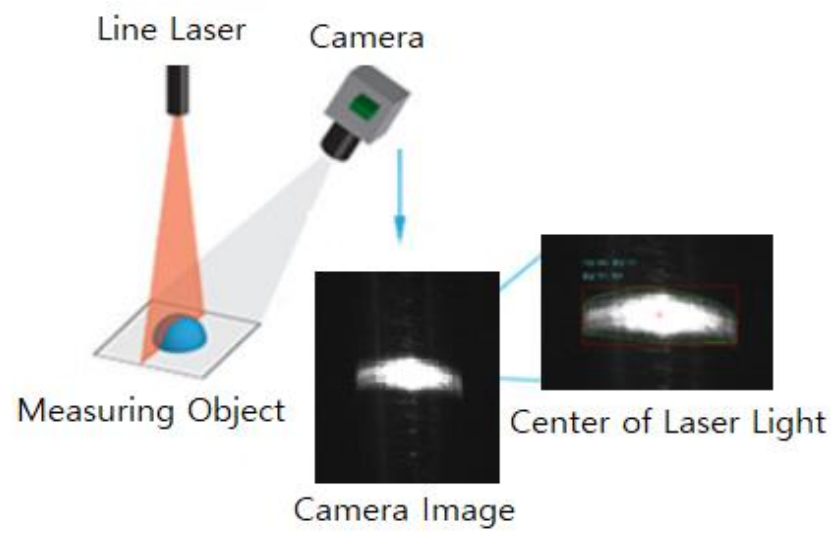

Figure 2. Methodology for measurement of pipe shape

\subsection{Vertex location}

Vertex location is calculated as point intersecting line A, which is connecting the two centers of pipe before bending, and line B, which is connecting the two centers of pipe after bending as Eq. (2).

$$
P^{\prime}=(x p, y p)=\left(x 1-\frac{x 2-x 1}{y 2-y 1} y 1,0\right)
$$

where, point $\mathrm{P}^{\prime}$ is a vertex location when $\mathrm{P}$ is considered as origin of coordinate, $\mathrm{xp}$ is a $\mathrm{x}$ directional coordinate of vertex location, and yp is a y-directional coordinate of vertex location and 0 .

\section{Configuration of pipe shape measurement head}

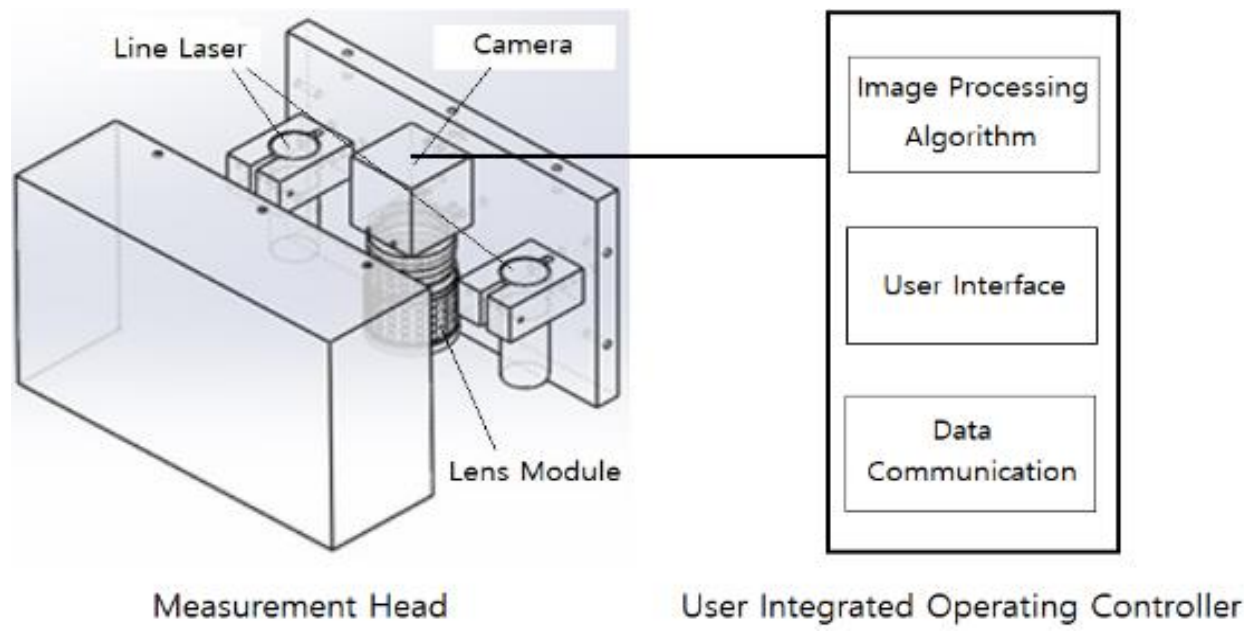

Figure 3. Configuration of pipe shape measurement system 
Pipe shape measurement head is consisted of two line-lasers and camera having lens module, and user integrated operating controller having image processing algorithm as shown in Fig. 3. Pipe image captured by camera is transferred to user integrated operating controller by Ethernet communication and is processed.

\section{Development and verification test of measurement head}

Two line-lasers having $650 \mathrm{~nm}$ wavelength and 300mm focal length, CMOS camera having 1.2 megapixel and giga Ethernet communication, and lens module having $25 \mathrm{~mm}$ focal length and 15.1"x20" field of view are used to construct the measurement head as shown in [Figure 4].

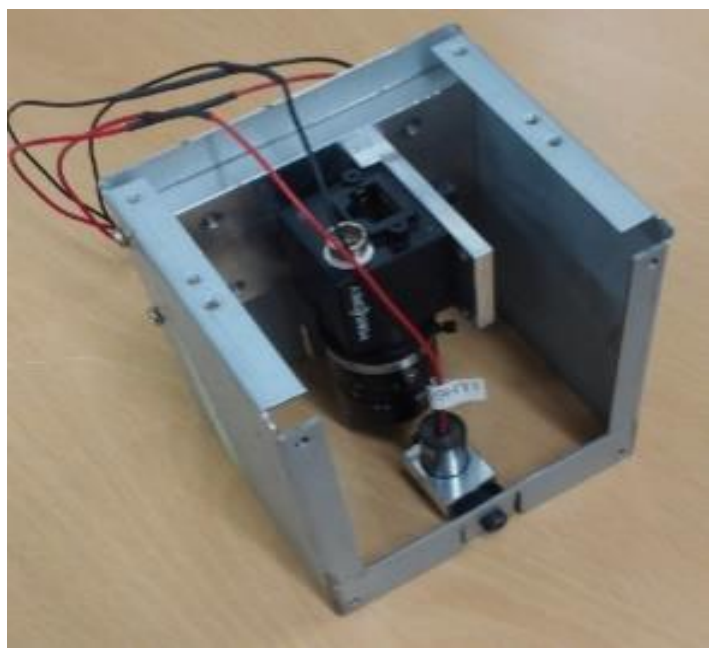

Figure 4. Developed real-time pipe shape measurement head

The specifications of developed measurement head are listed in Table 1. Measuring area of image is $96 \times 128 \mathrm{~mm}^{2}$. Two laser lights are separated $80 \mathrm{~mm}$ distance each other along the pipe.

Table 1. Specifications of developed measurement head

\begin{tabular}{|c|c|c|}
\hline Variable & Value & Unit \\
\hline Measuring resolution & 0.05 & $\mathrm{~mm}$ \\
\hline Measuring area & $96 \times 128$ & $\mathrm{~mm}^{2}$ \\
\hline Working distance & 300 & $\mathrm{~mm}$ \\
\hline
\end{tabular}

Experimental equipment for verification of the developed measurement head is constructed as shown in [Figure 5]. Pipe image is captured, transferred, and processed to calculate the center coordinate of pipe spotted by line laser. Figure 6 shows the captured and processed images of pipe. 


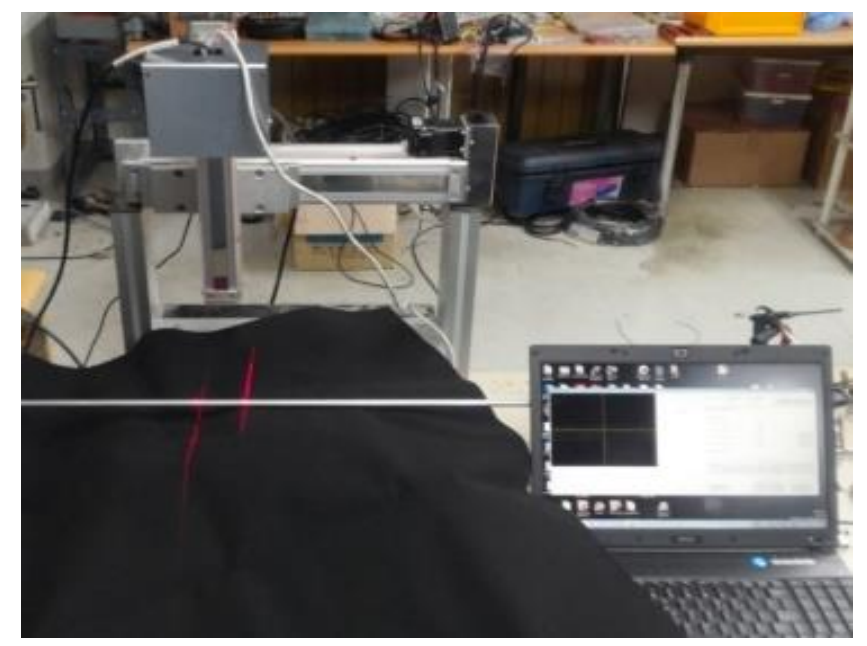

Figure 5. Experimental equipment for verification of the developed measurement head

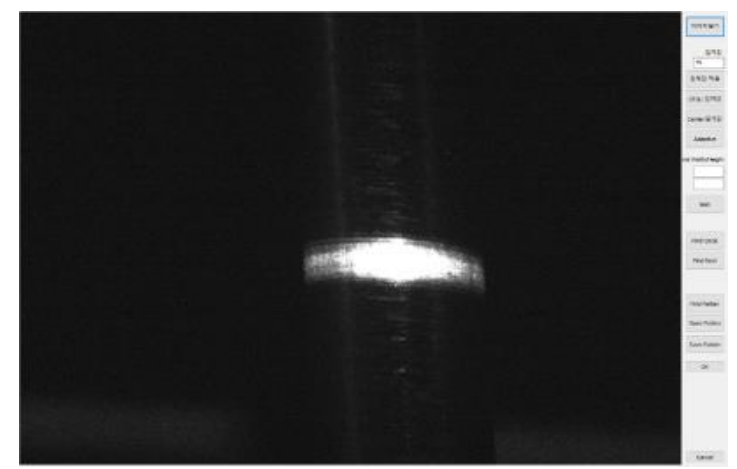

(a)

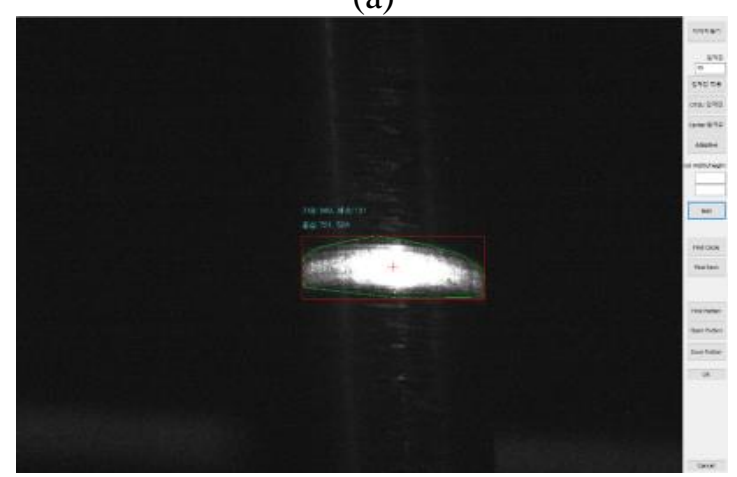

(b)

Figure 6. Image of pipe; (a) image captured by measurement head (b) image processed by image processing algorithm

The developed pipe shape measurement head is adopted in the pipe bending machine to verify the availability as shown in [Figure 7]. During the bending process, pipe image is captured by camera and pipe shape having bending angle and vertex location is calculated by image processing algorithm as shown in [Figure 8]. 


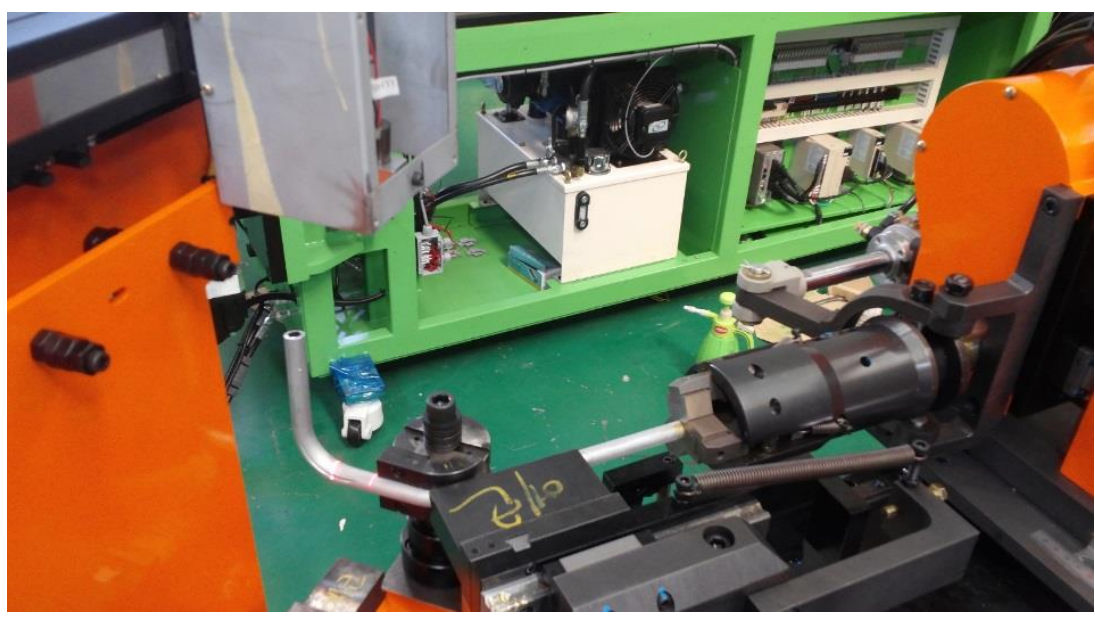

Figure 7. Developed measurement head adopted in the pipe bending machine

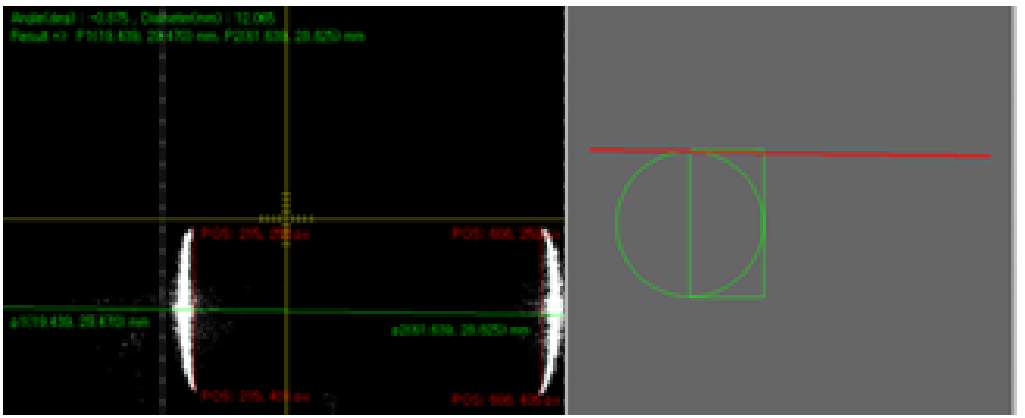

(a)

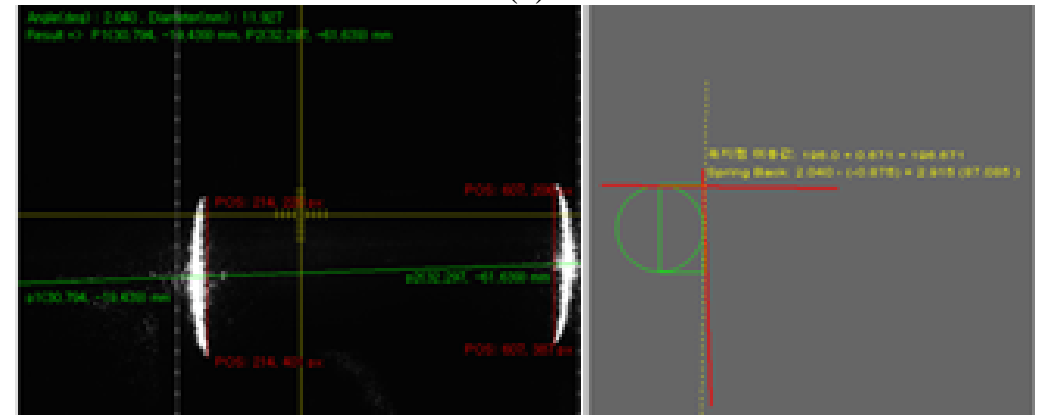

(b)

Figure 8 Captured pipe image and calculated pipe shape during bending process with bending angle of $90^{\circ}$; (a) before bending process, (b) after bending process

\section{Conclusions}

In this paper, head for real-time measurement of pipe shape proposed by using the analysis of change of pipe shape. Then, pipe shape measurement head constructed and developed. Also, developed pipe shape measurement head is adopted and tested in the pipe bending machine. From the study, we expect that the head for measurement of pipe shape is applicable during its bending process and avoidable faults during assembling.

In the future, we plan to evaluate the accuracy of the bending angle and the vertex location calculated by pipe shape measurement system. 


\section{Acknowledgements}

This work was supported by the National Research Foundation of Korea (NRF) grant funded by the Korea government (NRF-2018R1D1A1B07041829).

\section{References}

[1] Y. Hea, L. Henga, Z. Zhiyonga, Z. Meia, L. Jinga, and L. Guangjunb, “Advances and trends on tube bending forming technologies," Chinese Journal of Aeronautics, vol.25, no.1, pp.1-12, (2012)

[2] S. Zhang and J. Wu, "Spring back prediction of three-dimensional variable curvature tube bending," Advances in Mechanical Engineering, vol.8, no.3, pp. 1-13, (2016)

[3] H. J. Mun, C. S. Kim, and J. D. Kim, "A study on the development of the continuous 3D tube bending machine,” Trans. Korean Soc. Mech. Eng. A, vol.32, no.8, pp.701-706, (2008)

[4] J. W. Jeon, S. M. Lee, H. S. Jeong, and J. R. Cho, "Comparison of springback analysis and experiment for circular tube bending," Conference of Korean society of Marine Engineering, June 21; Korea, (2012)

[5] F. Song, H. Yang, H. Li, M. Zhan, and G. Li, "Springback prediction of thick-walled high-strength titanium tube bending," Chinese Journal of Aeronautics, vol.26, no.5, pp.1336-1345, (2013)

[6] Z. Guo and W. Tang, "Bending Angle Prediction model based on BPNN-spline in air bending springback process," Mathematical problems in engineering, pp.1-11, (2017)

[7] S. D. Mun, "Development of bending machine with high efficiency and precision forming," Journal of Korea Academia-Industrial Cooperation Society, vol.12, no.1, pp.7-14, (2011)

[8] M. J. Chung and C. Y. Kim, "Method for real time automatic measurement of pipe shape after bending process in the pipe bending machine," KSME 2016 Annual Meeting, December 14-16, Korea, (2016)

\section{Author}

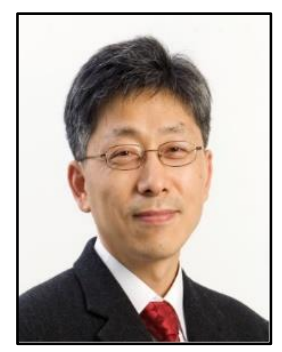

\section{Myung-Jin Chung}

1989: BS degree in Mechanical Engineering, Hanyang University.

1991: MS degree in Production Engineering, KAIST.

2002: PhD degree in Mechanical Engineering, KAIST.

1989 1997: Research Engineer, Hyundai Electronics.

1997 2004: Research Engineer, Hynix Semiconductor.

2004 current: Professor, Dept. of Mechatronics Engineering, Korea

Polytechnic University. 
The Study on the Real-Time Measurement of Pipe Shape Applicable During Its Bending Process

This page is empty by intention. 